\title{
The Challenges of Recommending Digital Selves in Physical Spaces
}

\author{
Joseph F. McCarthy \\ Nokia Research Center \\ 955 Page Mill Road \\ Palo Alto, CA 94304 USA \\ $+1.650 .804 .6987$ \\ joe.mccarthy@nokia.com
}

\begin{abstract}
There are a number of online systems where people can, in effect, recommend themselves, or more precisely, represent themselves in ways that may motivate others to seek them out for conversations, business ventures, dates and a variety of other types of interactions and relationships. Several of these systems offer capabilities for people to create profiles of themselves and algorithms for matching profiles to recommend one person to another. When a group of people is gathered together in a physical space, for the purpose of renewing connections or creating new ones, new challenges emerge with respect to which dimensions of people's online representations to inject into that space - how, where and when to recommend whom to whom. This paper briefly describes some experiences and ongoing challenges encountered in determining how best to bridge the gaps between people by bridging the gaps between people's online representations of themselves and their presence in physical space.
\end{abstract}

\section{Categories and Subject Descriptors}

H.5.3 [Information Interfaces and Presentation]: Group and Organization Interfaces - collaborative computing, computer supported cooperative work, synchronous interaction.

\section{General Terms}

Design, Economics, Experimentation, Human Factors

\section{Keywords}

Online social networking services, recommender systems, online profiles, physical spaces, proactive displays

\section{INTRODUCTION}

People gather together in a variety of times and places to meet new people with whom they might accomplish a variety of shared or related goals, such as discovering new ideas, finding potential romantic partners, or identifying business partners. Examples of events that facilitate these kinds of meetings include academic conferences, speed dating and business networking functions.

Permission to make digital or hard copies of all or part of this work for personal or classroom use is granted without fee provided that copies are not made or distributed for profit or commercial advantage and that copies bear this notice and the full citation on the first page. To copy otherwise, or republish, to post on servers or to redistribute to lists, requires prior specific permission and/or a fee.

RecSys '07, October 19-20, 2007, Minneapolis, Minnesota, USA.

Copyright 2007 ACM 978-1-59593-730-8/07/0010 _..\$5.00.
While many people may attend these kinds of events, finding the right people with whom one wants to connect - or finding the right topics about which to connect - can feel analogous to finding a needle in a haystack. A number of online systems have been created to help people create profiles for themselves, which are then used to recommend other people who may represent good matches (with respect to the goals of the events).

These systems can provide useful planning tools for those willing to spend the time to construct profiles ahead of time - and do the research before and during the event - to take advantage of the recommendations. An alternate approach is to require less initial investment by the prospective attendees, and to present more spontaneous and/or serendipitous opportunities for people to connect with one another during an event.

The following sections will describe a few examples of different approaches for recommending people to one another, especially systems that focus on connecting people at events they intend to attend, highlighting their relative advantages and disadvantages, and identifying some challenges for designing systems to recommend people to one another in shared physical spaces.

\section{PEOPLE RECOMMENDER SYSTEMS}

There are a number of online systems that are designed to support recommending people to each other electronically, for professional and/or personal objectives. Prominent examples include:

- $\quad$ LinkedIn (www.linkedin.com), which allows people to create professionally-oriented profiles of themselves, establish links to people they've collaborated with, and offer testimonials of others' work.

- Match.com (www.match.com), which allows people to create more personal profiles (or portraits) of themselves, and send electronic winks to people they'd like to connect with for romantic collaborations.

- MySpace (www.myspace.com) and Facebook (www.facebook.com) are more general online social networking services, that offer a variety of mechanisms for establishing and maintaining a variety of personal and professional relationships.

Each of these systems, and many others like them, offer platforms on which people can construct digital representations of themselves [Goffman, 1959]. Members of these sites can be seen as, in effect, promoting or recommending themselves, with varying degrees of intentionality. Although connections established through these online services may lead to or result 
from physical world interactions, much of their emphasis is in the online space.

There are a number of other systems whose primary focus is on establishing connections in physical spaces. These typically include some kind of online profile information, and the key is to use that information to promote physical world interactions. Examples include:

- introNetworks (www.intronetworks.com), offers an online tool, eventNet, for enabling prospective attendees of an event to specify keywords representing their interests, and a graphical interface for identifying people with similar interests, and mechanisms for contacting such people before the event. The SpotMe Conference Navigator (www.spotme.com) and nTag Interactive badge (www.ntag.com) offer mobile devices that enable on-site in-situ connections; Relescope [Farrell, et al., 2005] was a paper-based approach to recommending people with similar interests.

- One Key Away (www.onekeyaway.com) offers prospective attendees at a speed dating event the opportunity to answer a 64-question survey, compiles those answers into a vector that is downloaded into a MatchlinC keyfob-like device, which can then be used at the event to "zap" prospective dating partners to get a three mode (red, amber, green) indication of compatibility, reminiscent of the MemeTag system [Borovoy, et al., 1998] with its 7-element LEDs for indicating compatibility among attendees of a more professionally-oriented meeting.

- Proactive Displays ([McCarthy, et al., 2004], interrelativity.com/proactivedisplays) have been used to offer prospective attendees at a conference or other meeting the opportunity to create a profile, associate that profile with a radio frequency identification (RFID) tag, and then have elements of those profiles appear on a large display whenever the people associated with the profiles (and tags) are detected near that display.

\section{EXPERIENCES AND CHALLENGES}

Unfortunately, there have been relatively few reports of experiences with any of the abovementioned systems, although we are starting to see more studies of the design and impact of such systems (e.g., McCarthy, et al. [2004], Farrell, et al. [2005], Lampe, et al. [2007]). The remainder of this paper will identify a few of the challenges for any recommender system designed to use online profiles to recommend people to each other in shared physical spaces.

One of the most significant challenges is the implicit or explicit cost / benefit analysis performed by all prospective users of such a system. On the one hand, the richer the profiles, the better the recommendations, but time spent constructing profiles has to be balanced against time spent reaping the benefits of such profiles. For purely online systems, which persist over long periods of time, there is incentive for people to take the time to create rich profiles. However, for systems deployed for limited periods of time (e.g., an evening or a few days), there is less incentive, and so the profiles either have to be simpler and/or involve elements drawn from other, more persistent, sources of profile information.
In addition to the size and complexity of profiles, it is important to determine which online profile elements are most conducive to establishing connections between people. Many systems use keyword approaches for specifying interests. Others use people (typically represented by their names). Another approach is to use photos or images to reflect interests, creating situated affordances for object-centered sociality [Knorr Cetina, 1997]. One long-term informal longitudinal study of approachability and interactions was based on a profile that consisted of nothing more than a simple adhesive name tag [Ginsberg, 2003]

Another design criterion is the choice of recommendation conveyance device. Some systems require the conveyance of recommendations of potentially interesting people to take place outside the context of the event itself. Others use personal devices to convey recommendations, which typically occur in $1: 1$ interactions, which may be better suited to some settings (and goals) than others. Others use shared devices, such as large displays, that enable people to, in effect, recommend themselves to a larger co-located group within an event setting.

\section{CONCLUSION}

The proliferation of online profiles, personal digital devices and situated sensors and effectors in physical spaces (e.g., digital signage), creates ever-increasing opportunities for bridging the gaps between people by bridging the gaps between their online representations of themselves and their presence in shared physical spaces. Although this has not been a primary focus of recommender systems research, this is an area ripe for new approaches to help us connect more easily and effectively with the people around us.

\section{REFERENCES}

[1] Borovoy, Richard, Fred Martin, Sunil Vemuri, Mitchel Resnick, Brian Silverman and Chris Hancock. Meme Tags and Community Mirrors: Moving from Conference to Collaboration. Proc. of ACM Conf. on Computer Suppoted Cooperative Work (CSCW 1998). 159-168.

[2] Farrell, Stephen, Christopher Campbell, Suvda Myagmar. Relescope: An Experiment in Accelerating Relationships. Proc. of the ACM Conf. on Human Factors in Computing Systems (CHI 2005), Extended Abstracts. 1363-1366.

[3] Ginsberg, Scott. Hello, My Name is Scott. Front Porch Publishing. 2003.

[4] Goffman, Erving. The Presentation of Self in Everyday Life. Doubleday/Anchor, New York. 1959.

[5] Knorr Cetina, Karin. Sociality with Objects: Social Relations in Postsocial Knowledge Societies. Theory, Culture \& Society 14(4): 1-30. 1997.

[6] Lampe, Cliff, Nicole Ellison and Charles Steinfeld. A Familiar Face(book): Profile Elements as Signals in an Online Social Network. Proc. of ACM Conf. on Human Factors in Computer Systems (CHI 2007). 435-444.

[7] McCarthy, Joseph F., David W. McDonald, Suzanne Soroczak, David H. Nguyen and Al M. Rashid. Augmenting the Social Space of an Academic Conference. Proc. of the ACM Conf. on Computer Supported Cooperative Work (CSCW 2004), 39-48. 